\title{
The Gamma-Ray Imager/Polarimeter for Solar flares (GRIPS)
}

\author{
Albert Y. Shih*a ${ }^{\mathrm{a}}$, Robert P. Lin ${ }^{\mathrm{b}, \mathrm{c}, \mathrm{d}}$, Gordon J. Hurford ${ }^{\mathrm{b}}$, Nicole A. Duncan ${ }^{\mathrm{b}, \mathrm{c}}$, Pascal Saint-Hilaire ${ }^{\mathrm{b}}$, \\ Hazel M. Bain ${ }^{b}$, Steven E. Boggs ${ }^{b, c}$, Andreas C. Zoglauer ${ }^{b}$, David M. Smith ${ }^{\mathrm{e}}$, Hiroyasu Tajima ${ }^{\mathrm{f}}$, \\ Mark S. Amman ${ }^{\mathrm{g}}$, Tadayuki Takahashi ${ }^{\mathrm{h}}$

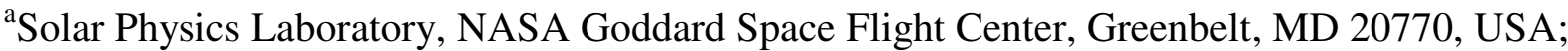 \\ ${ }^{\mathrm{b}}$ Space Sciences Laboratory, University of California, Berkeley, Berkeley, CA 94720, USA; \\ ${ }^{c}$ Department of Physics, University of California, Berkeley, Berkeley, CA 94720, USA; \\ ${ }^{\mathrm{d}}$ School of Space Research, Kyung Hee University, South Korea; \\ ${ }^{\mathrm{e}}$ University of California, Santa Cruz, Santa Cruz, CA 95064, USA; \\ ${ }^{\mathrm{f}}$ Solar-Terrestrial Environment Laboratory, Nagoya University, Nagoya, Japan; \\ ${ }^{\mathrm{g}}$ Lawrence Berkeley National Laboratory, Berkeley, CA 94720, USA; \\ ${ }^{\mathrm{h}}$ Institute of Space and Astronautical Science, Sagamihara, Kanagawa, Japan
}

\begin{abstract}
The balloon-borne Gamma-Ray Imager/Polarimeter for Solar flares (GRIPS) instrument will provide a near-optimal combination of high-resolution imaging, spectroscopy, and polarimetry of solar-flare gamma-ray/hard X-ray emissions from $\sim 20 \mathrm{keV}$ to $>\sim 10 \mathrm{MeV}$. GRIPS will address questions raised by recent solar flare observations regarding particle acceleration and energy release, such as: What causes the spatial separation between energetic electrons producing hard $\mathrm{X}$-rays and energetic ions producing gamma-ray lines? How anisotropic are the relativistic electrons, and why can they dominate in the corona? How do the compositions of accelerated and ambient material vary with space and time, and why? The spectrometer/polarimeter consists of sixteen 3D position-sensitive germanium detectors (3D-GeDs), where each energy deposition is individually recorded with an energy resolution of a few keV FWHM and a spatial resolution of $<0.1 \mathrm{~mm}^{3}$. Imaging is accomplished by a single multi-pitch rotating modulator (MPRM), a $2.5-\mathrm{cm}$ thick tungstenalloy slit/slat grid with pitches that range quasi-continuously from 1 to $13 \mathrm{~mm}$. The MPRM is situated 8 meters from the spectrometer to provide excellent image quality and unparalleled angular resolution at gamma-ray energies (12.5 arcsec FWHM), sufficient to separate $2.2 \mathrm{MeV}$ footpoint sources for almost all flares. Polarimetry is accomplished by analyzing the anisotropy of reconstructed Compton scattering in the 3D-GeDs (i.e., as an active scatterer), with an estimated minimum detectable polarization of a few percent at $150-650 \mathrm{keV}$ in an X-class flare. GRIPS is scheduled for a continental-US engineering test flight in fall 2013, followed by long or ultra-long duration balloon flights in Antarctica.
\end{abstract}

Keywords: solar flares, particle acceleration, balloon instrument, high-resolution gamma-ray imaging, high-resolution gamma-ray spectroscopy, gamma-ray polarimetry, germanium detectors

\section{INTRODUCTION}

The Sun is the most energetic particle accelerator in the solar system, producing ions up to $10 \mathrm{~s}$ of $\mathrm{GeV}$ and electrons to $100 \mathrm{~s}$ of $\mathrm{MeV}$, in both large solar flares and fast coronal mass ejections (CMEs), but through different physical mechanisms. Solar flares are the most powerful explosions in the solar system, releasing up to $10^{32}-10^{33}$ ergs in $100-$ 1,000 seconds, most likely through magnetic reconnection, with up to $\sim 10-50 \%$ of this energy in flare-accelerated electrons and a comparable amount in flare-accelerated $>\sim 1 \mathrm{MeV}$ ions ${ }^{1,2}$. The intense solar energetic particle (SEP) events directly observed in the interplanetary medium, however, appear to be accelerated by shock waves driven by fast CMEs, although there is almost always an associated large flare.

*albert.y.shih@nasa.gov; phone 1-301-286-1843 
High-energy emissions are the most direct signature of the acceleration of electrons, protons and heavier ions in solar flares. The hard X-ray and gamma-ray continuum is produced as bremsstrahlung by energetic electrons. Nuclear collisions of energetic ions with the ambient solar atmosphere result in a complex spectrum of narrow and broad gammaray lines that contain unique information on not only the accelerated ions but also the ambient solar atmosphere. The RHESSI (Reuven Ramaty High-Energy Solar Spectroscopic Imager) mission ${ }^{3}$ launched in 2002 provided a new window on particle acceleration at the Sun through imaging spectroscopy of the hard X-ray/gamma-ray continuum and gammaray line emission produced by the accelerated electrons and ions, respectively.

The balloon-borne Gamma-Ray Imager/Polarimeter for Solar flares (GRIPS) instrument is the next step in studying high-energy ion and electron acceleration in solar flares. GRIPS is part of the NASA Heliophysics Low Cost Access to Space program and will provide a near-optimal combination of high-resolution imaging, spectroscopy, and polarimetry of solar-flare gamma-ray/hard X-ray emissions from $\sim 20 \mathrm{keV}$ to $>\sim 10 \mathrm{MeV}$ (see Table 1). The PI of GRIPS is Robert P. Lin, and the project is a collaboration between the University of California, Berkeley; NASA Goddard Space Flight Center; the University of California, Santa Cruz; Nagoya University; Lawrence Berkeley National Laboratory; and ISAS. GRIPS is scheduled for a continental-US engineering test flight in fall 2013, followed by long- or ultra-longduration balloon flights in Antarctica. In addition to the science return from these flights, the technologies used by GRIPS will be proven for use on a spacecraft platform.

Table 1. Comparison of GRIPS and RHESSI capabilities

\begin{tabular}{|c|c|c|c|}
\hline & RHESSI & $\begin{array}{l}\text { GRIPS } \\
\text { (balloon) }\end{array}$ & $\begin{array}{l}\text { GRIPS-like } \\
\text { spacecraft }\end{array}$ \\
\hline Effective area at $2.2 \mathrm{MeV}$ & $\sim 3 \mathrm{~cm}^{2 *}$ & $\sim 13 \mathrm{~cm}^{2 * *}$ & $\sim 52 \mathrm{~cm}^{2}$ \\
\hline Effective area at $300 \mathrm{keV}$ & $\sim 50 \mathrm{~cm}^{2}$ & $\sim 55 \mathrm{~cm}^{2 * *}$ & $\sim 210 \mathrm{~cm}^{2}$ \\
\hline Effective area at $50 \mathrm{keV}$ & $\sim 47 \mathrm{~cm}^{2}$ & $\sim 89 \mathrm{~cm}^{2 * *}$ & $\sim 356 \mathrm{~cm}^{2}$ \\
\hline Angular resolution at $2.2 \mathrm{MeV}$ & $35^{\prime \prime}$ & $12.5 ”$ & $5 "$ \\
\hline $\begin{array}{l}\text { Minimum detectable polarization at } 150-650 \mathrm{keV} \\
\text { (for the } 2002 \text { July } 23 \text { flare), three-sigma level }\end{array}$ & $\sim 30 \%^{* * *}$ & $\sim 3 \%$ & $\sim 1 \%$ \\
\hline
\end{tabular}

\section{SCIENTIFIC OBJECTIVES}

GRIPS will address questions raised by recent solar-flare observations by RHESSI and other missions regarding particle acceleration and energy release, such as:

- What causes the spatial separation between energetic electrons producing hard X-rays and energetic ions producing gamma-ray lines?

- How anisotropic are the relativistic electrons, and why can they dominate in the corona?

- How do the compositions of accelerated and ambient material vary with space and time, and why?

Energetic protons and alpha particles colliding with ambient carbon and heavier nuclei produce narrow prompt deexcitation lines (widths of a few $\mathrm{keV}$ to $\sim 100 \mathrm{keV}$ ), while energetic heavy nuclei colliding with ambient hydrogen and helium produce much broader lines due to Doppler broadening (widths of a few hundred $\mathrm{keV}$ to an $\mathrm{MeV}$ ). These nuclear collisions also produce positrons and neutrons. The positrons annihilate with electrons to produce a line at $0.511 \mathrm{MeV}$, while the neutrons first thermalize and are then captured by protons to produce a line at $2.223 \mathrm{MeV}$, usually the strongest line $^{5}$. RHESSI has provided the first high-resolution gamma-ray spectroscopy ${ }^{6}$ and the first gamma-ray line imaging of solar-flare emission?

Gamma-ray line imaging provides the only way to directly observe the spatial properties of flare-accelerated ions near the Sun. The first such image was obtained for the 2002 July 23 flare in the $2.223 \mathrm{MeV}$ line ${ }^{7}$. The spatial centroid of the 2.223 MeV line source was found to be displaced from the corresponding centroid of the electron-bremsstrahlung 
continuum emission by $\sim 15,000 \mathrm{~km}$, a very surprising result given the general similarity of the time profiles of electronassociated X-ray and ion-associated gamma-ray line emissions. Subsequently RHESSI imaged three flares in the OctNov 2003 series of X-class flares ${ }^{8}$. For all these events, the accelerated $>\sim 10 \mathrm{MeV}$ ions, as evidenced by the $2.223 \mathrm{MeV}$ line sources, are interacting in the vicinity of the flare, with most, if not all, of the emission spatially confined to compact sources with upper limits to the size of a few tens of arcseconds. These results indicate that the gamma-ray-producing ions are accelerated in the flare itself and not by a CME shock wave or other process that extends over a large portion of the Sun.

The RHESSI gamma-ray line images are severely limited by poor spatial resolution (the two rotating modulation collimators capable of gamma-ray imaging provide 3-arcmin and 35-arcsec resolution, and their point-response functions have large sidelobes), as well as by low sensitivity. Of the flares observed by RHESSI, only the 2003 Oct 28 flare was large enough so that two footpoints, separated by $\sim 80$ arcsec, could be detected in the $2.223 \mathrm{MeV}$ line image (Figure 1), straddling the arcade of loops in the TRACE image. This key observation of two ion footpoints strongly suggests that ion acceleration, like electron acceleration, is closely associated with magnetic reconnection. The hard Xray, $\sim 0.2-0.3 \mathrm{MeV}$ electron-bremsstrahlung image also showed two footpoint sources straddling the arcade, but they are displaced by 14 and $17 \pm 5$ arcseconds from the $2.223 \mathrm{MeV}$ sources, especially surprising since the $>0.3 \mathrm{MeV}$ hard X-ray fluence shows a close linear correlation to the $2.223 \mathrm{MeV}$ fluence over more than three orders of magnitude ${ }^{9}$. At present, the displacement of the ion sources from the electron sources, seen in both flares where the measurements can be made, are unexplained. Gradient and curvature drifts in the arcade magnetic fields are inadequate by $>\sim$ two orders of magnitude.

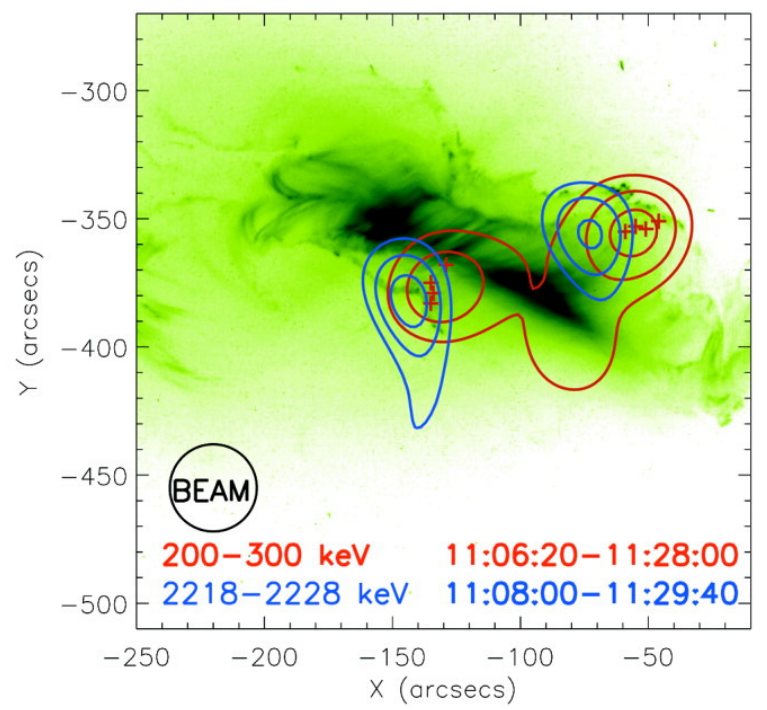

Figure 1. RHESSI 2.223 MeV gamma-ray line images (blue contours, 35-arcsec resolution) made with RMCs 6+9 (50, 70 and $90 \%$ contours) overlaid on a TRACE $195 \AA$ image of the 2003 October 28 flare. The red contours are $200-300 \mathrm{keV}$ bremstrahlung X-rays (note the coronal source between the footpoints); "+" symbols indicate footpoint locations for shorter successive integrations of 100,120,180 and 240 seconds beginning at 11:06:20 UT. The X and Y heliographic offsets are positive West and North of Sun center.

It is of great scientific interest to obtain higher spatial resolution and more sensitive gamma-ray line imaging, and to extend this to other lines such as the positron annihilation line at $0.511 \mathrm{MeV}$, the prompt de-excitation lines, and the broad lines. The positron annihilation line is an excellent diagnostic of the temperature and density in the annihilation region. RHESSI high-resolution spectroscopy resolved this line for the first time, showing that it has a typical width of 5-8 keV FWHM, implying temperatures of several hundred thousand degrees ${ }^{10}$. To stop the positrons, a substantial amount of plasma must exist at this temperature in the flaring atmosphere, well beyond what exists in the quiet atmosphere. Positron-annihilation line observations thus provide a probe into a plasma component not otherwise accessible. In the 2003 October 28 flare, the line was observed to narrow suddenly to about $1 \mathrm{keV}$ in width - the narrowest ever observed in astrophysics. One possible explanation is that the source has moved to a much cooler region. Imaging with temporal resolution would clearly provide crucial information about this line. 
RHESSI's gamma-ray imaging capabilities have also been used to study the gamma-ray continuum emission produced by accelerated electrons. In three large flares observed by RHESSI, imaging shows emission in the 200-800 keV range, not only from the footpoints of flare loops, but also strong emission from a source in the corona ${ }^{11}$ (see also Figure 1). The coronal sources show spectra with harder spectral indices than the footpoints, which suggest that higher energy electrons may remain trapped in the corona while lower energy electrons escape and lose their energy at the footpoints. Images of these coronal sources are limited by counting statistics as well as the point response of the imager.

Furthermore, the polarization of this emission is a powerful diagnostic of electron beaming in solar flares, and the direction of the polarization vector - whether parallel or perpendicular to the projected magnetic field - will depend on whether the electrons are primarily beamed along the magnetic field lines (small pitch angle) or perpendicular (large pitch angle). Solar X-ray polarization measurements have historically proved difficult ${ }^{12}$, and have mainly been at energies below $20 \mathrm{keV}$ where the emission is primarily thermal. Polarization studies ${ }^{13}$ using the modulation produced by a passive beryllium scatterer next to the RHESSI detectors suggest that hard X-ray emission from the 2002 July 23 flare might be polarized at energies of $\sim 20-40 \mathrm{keV}$. The best energy range for studying flare polarization is $>50 \mathrm{keV}$ and below $\sim 1 \mathrm{MeV}^{14,15}$. At these higher energies, the polarization is expected to be stronger since there is relatively less scattering to reduce any beaming. Above $\sim 150 \mathrm{keV}$, Compton scattering dominates over photoelectric absorption in germanium, and the RHESSI detectors can be analyzed as active scatterers via detector-detector coincidences. Marginal $(<2 \sigma)$ detections of polarization at gamma-ray energies $(0.2-1 \mathrm{MeV})$ have been reported for some large flares ${ }^{4,16}$. These measurements hint at the possibility of obtaining useful constraints on magnetic-field topologies and acceleration models, but much better measurements are required.

\section{INSTRUMENT DESCRIPTION}

The key new technology used by GRIPS is a 3D position-sensitive germanium detector (3D-GeD) that allows the position and energy deposition of every photon interaction to be recorded individually, even within the same detector. With the 3D-GeDs, GRIPS can use a new single-grid imaging system, called the multi-pitch rotating modulator (MPRM), that provides a near-ideal point response function with twice the throughput per $\mathrm{cm}^{2}$ of detector area compared to the rotating modulation collimator (RMC) imaging of RHESSI.

The balloon-borne GRIPS instrument consists of a spectrometer/polarimeter with sixteen 3D-GeDs (Figure 2), together with a single-grid MPRM imaging system on a boom 8 meters away. The 3D-GeDs provide a spatial resolution of $\sim 0.5$ $\mathrm{mm}$, and energy resolution of a few keV FWHM (typical for cryogenically cooled germanium detectors) from $\sim 20 \mathrm{keV}$ to $>\sim 10 \mathrm{MeV}$. The MPRM provides a full-Sun field-of-view, high sensitivity, excellent image quality, and angular resolution (12.5-arcsec FWHM at gamma-ray energies) sufficient to separate the footpoints of $2.2 \mathrm{MeV}$ sources for all large flares; the electron footpoint separations of all RHESSI gamma-ray sources imaged to date range from 21 to 77 arcseconds, with a median value of 33 arcseconds. At $2.2 \mathrm{MeV}$, RHESSI's angular resolution is only 35 arcseconds.

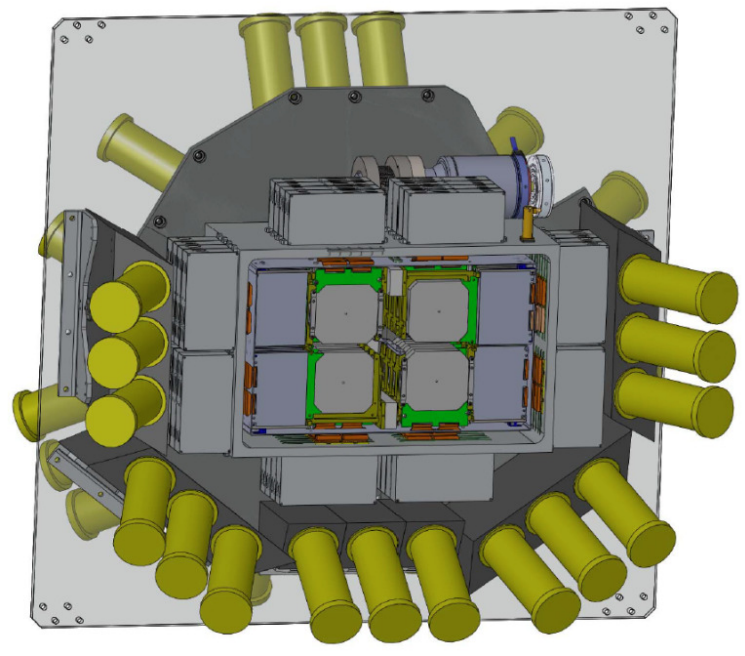

Figure 2. Model of the GRIPS spectrometer/polarimeter consisting of sixteen 3D-GeDs in a single cryostat, surrounded by the BGO shield (with PMTs). Parts of the structure are shown as transparent for visibility. 
The sixteen 3D-GeDs in the spectrometer/polarimeter are arranged in four $2 \times 2$ planes and housed in a single cryostat, with associated ASIC electronics immediately outside the cryostat wall. To reduce background, the cryostat is surrounded by a 5 -cm thick bismuth germanate (BGO) scintillator active anti-coincidence shield read out by 36 individual PMTs. With a threshold of $80 \mathrm{keV}$, the BGO shields significantly reduce the instrumental background even before the Compton imaging and the spectral signature techniques are applied.

\subsection{D position-sensitive germanium detectors (3D-GeDs)}

The GRIPS 3D-GeDs are fabricated by Lawrence Berkeley National Laboratory (LBNL). Compared to the earlier generation of 3D-GeDs that have already flown on the Nuclear Compton Telescope (NCT) balloon instrument ${ }^{16}$, the GRIPS 3D-GeDs have the same $\sim 75-\mathrm{mm} \times 75-\mathrm{mm} \times 15-\mathrm{mm}$ thick planar geometry, but with orthogonal 0.5-mm pitch electrode strips on opposite sides (versus NCT's 2-mm pitch).

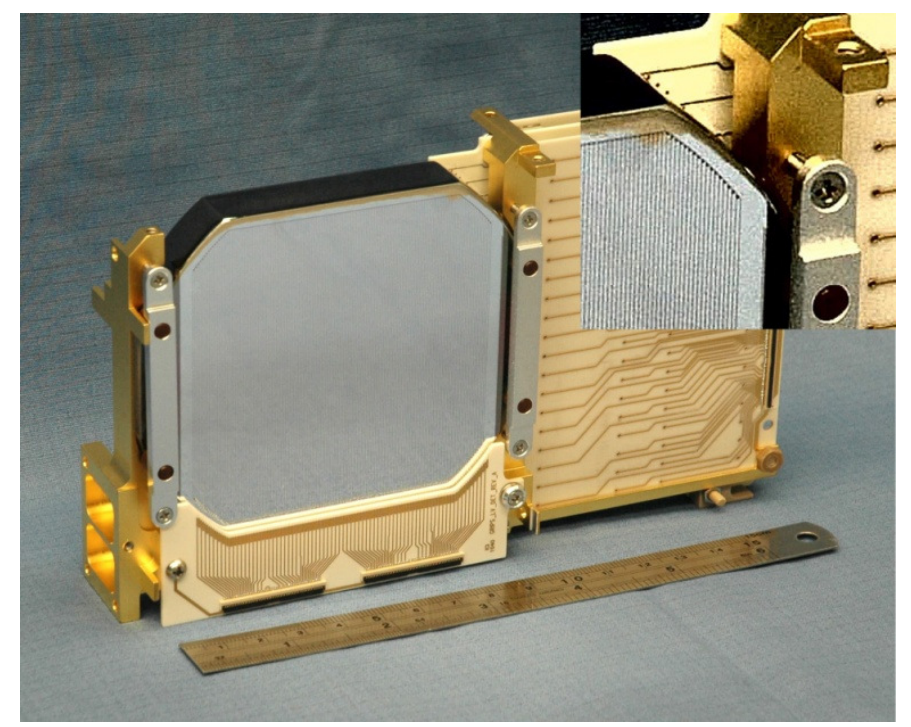

Figure 3. A GRIPS 3D-GeD, with a 54- $\mathrm{cm}^{2}$ active area, a $15-\mathrm{mm}$ thickness, and $0.5-\mathrm{mm}$ pitch strips on each face (shown zoomed and in high contrast in inset)

3D position resolution: These extremely thin strips, combined with signal timing, provide full 3D position resolution to $<0.1 \mathrm{~mm}^{3}$. Whenever a photon interacts in a GeD, by photoabsorption or Compton scattering, a fast recoil electron is produced which knocks more electrons from the valence band to the conduction band, leaving holes behind. In an applied electric field ("bias voltage") these e-h pairs will drift in opposite directions, electrons toward the anode and holes toward the cathode. By segmenting the anode into $0.5-\mathrm{mm}$ pitch strips, and the cathode into orthogonal $0.5-\mathrm{mm}$ pitch strips, 2D positioning is achieved directly through identification of the active anode and cathode strips.

The e-h pairs have finite drift velocities, $\sim 0.1 \mathrm{~mm} / \mathrm{ns}$. Positioning in the third dimension is achieved directly by the time difference between the electron collection and the hole collection on opposite faces of the detector ${ }^{18}$. Amrose et al. ${ }^{19}$ showed that anode-cathode collection times provide a robust, nearly linear depth measurement. NCT detectors consistently reach a depth resolution of $\sim 0.4 \mathrm{~mm}$ FWHM for all interaction energies $>40 \mathrm{keV}$.

GeD fabrication: The 3D-GeDs operate as fully-depleted diodes and are produced using the amorphous semiconductor electrical contact technology pioneered by $\mathrm{LBNL}^{20}$. The blocking electrodes are made from a $\sim 0.1-\mu \mathrm{m}$ thick layer of amorphous $\mathrm{Ge}$ (or $\mathrm{Si}$ ) which is deposited on the entire detector surface. The strip electrodes are simply defined by a patterned metal film deposited on top of the amorphous Ge film. The amorphous Ge film fully passivates every detector surface not used for electrical connection. The bipolar blocking behavior of the amorphous contacts allows them to be used on both sides of the detector, thereby replacing the conventional n-type lithium contact and p-type ion-implanted contact. The amorphous Ge can also be made highly resistive which provides excellent inter-electrode impedance ${ }^{21}$ and allows excellent spectroscopic performance to be achieved ${ }^{17}$. Since our 3D-GeDs are cut from a single homogeneous Ge crystal, their efficiency, spectral resolution, and position resolution are uniform across the face of the detector ${ }^{22}$. Variations between strips are generally on the few-percent level, and are easily calibrated. 
Spectral performance: Our 3D-GeDs maintain the high spectral performance characteristic of Ge detectors. There is some charge loss $(\sim 1 \%$ maximum) for events shared between strips (i.e. interactions that occur in the gaps between strips). A gap of $0.06 \mathrm{~mm}$ between the strips was chosen to optimize the spectral resolution $(\sim 1.8 \mathrm{keV}$ FWHM at 662 $\mathrm{keV}$ ) - a smaller gaps lowers charge loss resulting from charge collection to the gap area, whereas a larger gap size lowers the strip capacitance and the associated electronics noise. From the ratio of the charge collected on the two neighboring strips, we can correct the measured energies for the shared events to regain most of the original resolution ${ }^{22}$. In addition, the interaction energies are measured redundantly on the two faces of the detector, minimizing reliance on these correction techniques.

Compton Techniques: These 3D-GeDs can use the physics of Compton scattering to benefit spectroscopy, imaging, and polarimetry. When an incoming photon undergoes a Compton scatter at an angle with respect to its initial direction at the position, it creates a recoil electron that induces the signal measured in the detector. For a given initial photon energy and recoil electron energy, the scattering angle can be uniquely determined. The scattered photon then may undergo a series of one or more interactions, which are also measured. If a given photon Compton scatters several times in the detector, then its track can be reconstructed, not only to provide the full initial energy, but also to constrain its incident direction to within an annulus in the $\mathrm{sky}^{23,24}$. While the Compton imaging resolution $\left(\sim 2^{\circ}\right)$ is too crude for solar imaging, it can drastically reducing the background to only those events consistent with a solar origin. Compton techniques can also isolate which interaction site was the first, thus allowing the 3D-GeDs to act similar to a pixilated array despite the successive scatters in the rest of the detector volume. Furthermore, since Compton techniques can reconstruct the initial photon energy given enough scattering sites even if the photon escapes the detector at some point and does not deposit its full energy, it is possible to decrease the amount of Compton continuum below a gamma-ray line and perhaps even increase the photopeak efficiency of the detector.

Polarization: For a linearly polarized incident photon, the scattering will preferentially scatter perpendicular to its direction of polarization. Since the $3 \mathrm{D}-\mathrm{GeD}$ array can detect the direction of a Compton scatter, a partially linearly polarized beam of photons will show a modulation of secondary interactions that depends on the scattering direction, and the amplitude of this modulation will be related to the level of polarization (Figure 4). Polarized photons that scatter at $\sim 90^{\circ}$ will show the greatest amount of modulation, and the compact design and high spatial resolution of the $3 \mathrm{D}-\mathrm{GeD}$ array maximizes the efficiency of catching such scatters.

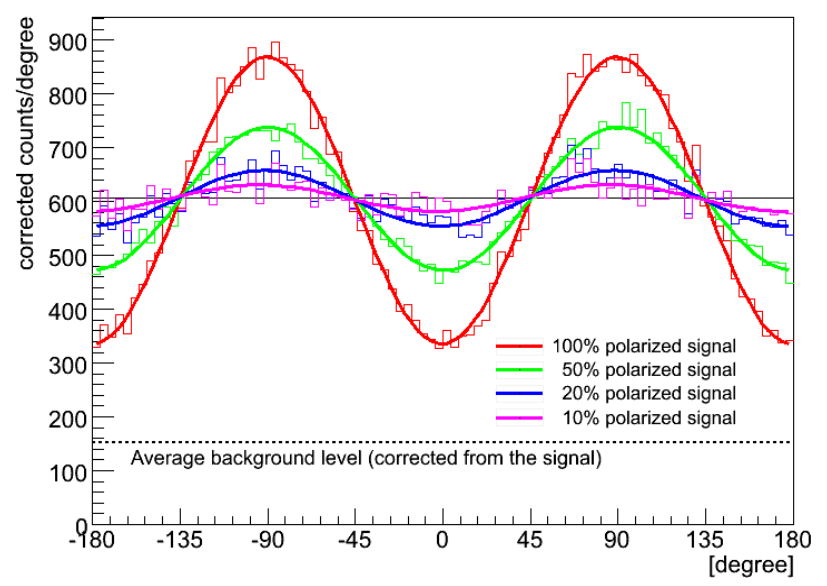

Figure 4. Simulated modulation of counts over 600 seconds from the X4.8 flare on 2002 July 23, for various levels of linear polarization, as would be observed by the GRIPS instrument on an Antarctic flight.

\subsection{D-GeD associated electronics}

Each 3D-GeD strip requires a compact, low power signal processing chain. Because of the large number of strips $(\sim 300$ per 3D-GeD), we use an application-specific integrated circuit (ASIC) with 64 channels, each with a charge-sensitive preamplifier, a slow shaper for energy measurements, and a fast shaper and comparator for timing measurements. The particular ASIC we use is the VATA 453 by Gamma-Medica Ideas, which was developed from an existing ASIC design developed by the KIPAC/ISAS team for the NeXT mission in Japan ${ }^{25}$, to provide the timing needed for GRIPS (Table 2). 
The VATA employs a constant fraction discriminator to minimize the time walk due to the pulse height and can meet the specification by optimizing the shaping time of the fast shaper. Another modification involves optimizing the front-end FET for noise performance for the larger capacitance load of the 3D-GeD strips.

Table 2. Summary of ASIC requirements

\begin{tabular}{|l|l|}
\hline $\begin{array}{c}\text { ENC (equivalent noise charge) for 35 } \mathrm{pF} \\
\text { input load, 50 pA leak current }\end{array}$ & $\begin{array}{c}360 \text { electrons } \\
\text { (RMS) }\end{array}$ \\
\hline Shaping time (slow shaper) & $\sim 4 \mu \mathrm{s}$ \\
\hline Trigger timing resolution & $10 \mathrm{~ns}(\mathrm{RMS})$ \\
\hline Minimum trigger threshold & $\sim 15 \mathrm{keV}(\mathrm{Ge})$ \\
\hline Power consumption & $20 \mathrm{~mW} /$ channel \\
\hline
\end{tabular}

The energy output of each ASIC channel is converted to a digital value in a Wilkinson ramp A/D converter, where the ramp is actually internal to the ASIC. External to the ASICs are FPGAs for timing measurements and control of the acquisition process, and a low power DSP to control the overall detector data channels, compact the data, and communicate with the rest of the instrument (Figure 5). Comparing the timing of channels on opposite sides of the detector provides the location of the interaction within the detector. Fast counters in the FPGA determine this relative time based on the trigger signals. This approach has been previously demonstrated to be successful in the NCT and is a low-power time to digital conversion.

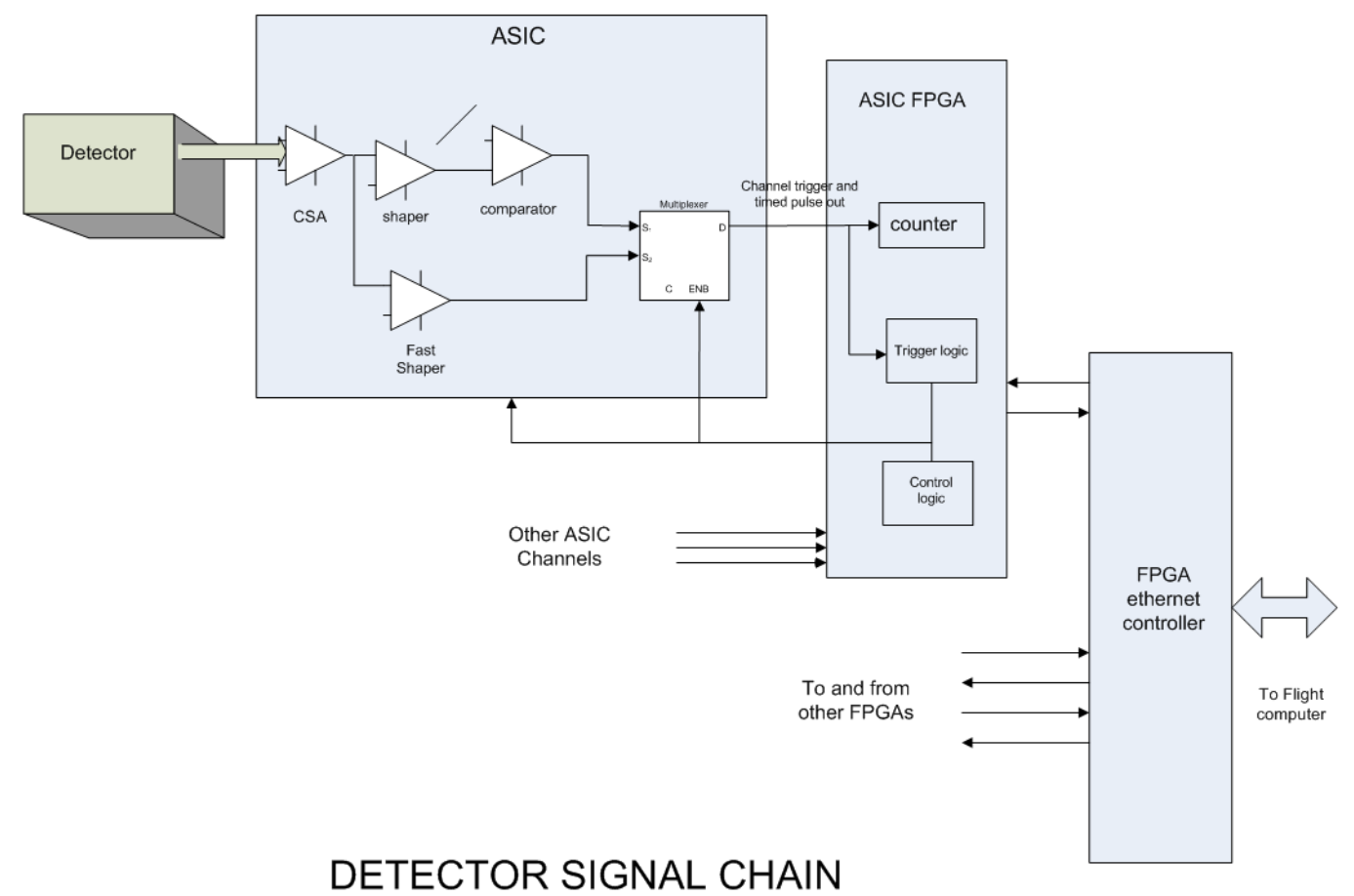

Figure 5. Representation of the data acquisition channels.

\subsection{Cryogenic cooling}

The single-vacuum cryostat is cooled by a single Stirling-engine cryocooler, similar to RHESSI, but with a more advanced Sunpower cryocooler. Many of the technologies developed for the RHESSI cryocooled spectrometer are being utilized. Each 3D-GeD is enclosed in its own carrier bracket, mounted on a central copper coldplate that is suspended on fiberglass bands to provide both rigid structural support and a high degree of thermal conductive isolation. The entire assembly is enclosed in multiple concentric thin IR radiation shields to minimize radiative coupling with the warm cryostat housing. Cryocooler waste heat is radiated from a silver-Teflon coated aluminum radiator looking skyward and away from the Sun. 


\subsection{Multi-pitch rotating modulator (MPRM) imager}

Focusing optics are not a viable option for these energies and angular resolutions. GRIPS imaging uses a new modulation technique, the "Multi-Pitch Rotating Modulator" (MPRM), for which the enabling technology is the high spatial resolution of the detector system. The MPRM shares the two primary advantages of the uniformly redundant array (URA) technique widely used in astrophysics: $50 \%$ throughput for high sensitivity and a point response function virtually free of sidelobes. However, the MPRM has the significant advantage, not shared by URAs, in that it is well suited to characterizing sources over a wide range of angular scales, a feature essential for solar flare studies.

For GRIPS, the MPRM consists of a single rotating $(15 \mathrm{rpm})$ planar grid located $8 \mathrm{~m}$ in front of the detector. The single grid consists of a large number of parallel tungsten/copper slats. If the pitch of the grid slats were uniform, then a detector able to spatially resolve the rotating X-ray shadow of the grid would enable the grid-detector combination to act as a rotating modulation collimator (RMC) with FWHM angular resolution $=0.5 *$ grid pitch / grid-to-detector separation. Since there is only one grid, however, the throughput would be twice that of the corresponding bi-grid RMC (50\% vs. $25 \%$ ), with an obvious gain in sensitivity. Imaging information is encoded in the time and spatial variations of the detected X-ray flux: at any instant, the X-ray flux varies spatially across the detector; at any location, the flux is timemodulated. The modulator's combination of time and spatial modulation is particularly robust against systematic errors. Ground-based software used reconstruct the image is based on techniques well-established with RHESSI ${ }^{26}$ (Figure 6).

In an MPRM this concept is extended by using a grid that has a quasi-continuous range of pitches instead of a uniform pitch. For photons incident on any given area of the grid, the system functions as an RMC with an angular resolution corresponding to the local grid pitch. But because of the range of grid pitches, the overall system measures a corresponding range of angular frequencies. With grid pitches ranging from 1 to $13 \mathrm{~mm}$ and a grid-detector separation of $8 \mathrm{~m}$, the GRIPS FWHM angular resolution runs quasi-continuously from 12.5 to 162 arcsecond in a manner that is tailored to optimize angular frequency weighting and imaging performance.

As the grid rotates, these angular frequencies are also measured at a continuous range of orientations. The result is that the MPRM has quasi-continuous, two-dimensional coverage in the spatial frequency (uv) plane. The resulting image quality represents a substantial improvement over that possible on RHESSI (Figure 6 and Table 3).

(a)

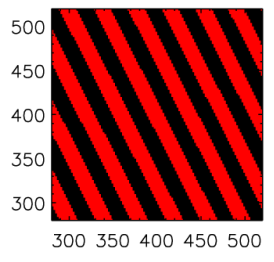

(d)

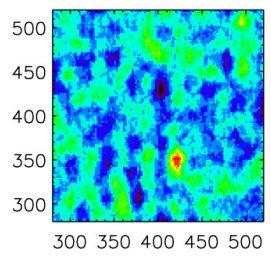

(b)

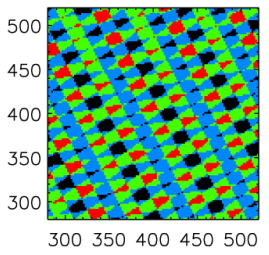

(e)

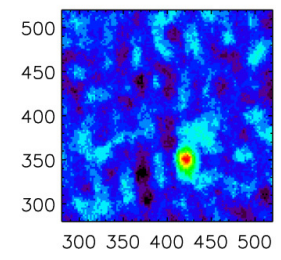

(c)

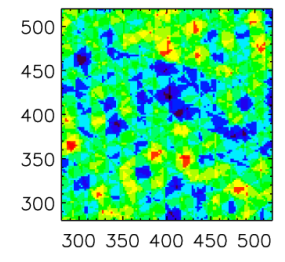

(f)

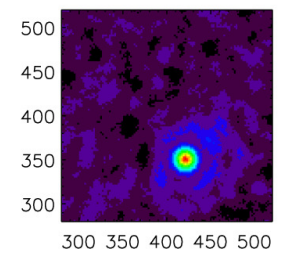

(g)

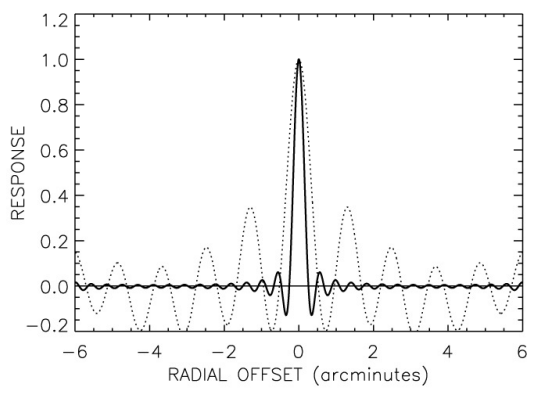

Figure 6. Schematic illustration of GRIPS image reconstruction. To form an image, detector-specific algorithms are first used to identify the subset of events that correspond to the user's energy, time and/or polarization criteria. For imaging, these events are characterized by their location and time (x,y,t). For each such event, aspect and grid orientation knowledge is used to form a 'striped' back-projected probability map of the desired field of view on the Sun showing possible directions from which this photon could have originated (Panel a). Subsequent events add additional 'stripes' of differing orientations, widths and phases. Panels a-f correspond to 1, 3, 10, 30, 100 and 1000 counts from a simulated point source, respectively. It shows that a source can be reliably located with as few as $\sim 100$ counts. For more complex sources, the backprojected map represents a convolution of the source with the circularly-symmetric point response function. Such a "dirty map' can then be improved with algorithms such as CLEAN. A similar "Back Projection + CLEAN" process forms the basis of RHESSI image reconstruction. The radial profile for the GRIPS point response function (Panel $\mathrm{g}$, black curve) is much improved over the corresponding RHESSI point response function for gamma rays (dotted curve). 
Table 3. Comparison of GRIPS and RHESSI imaging techniques

\begin{tabular}{|c|c|}
\hline GRIPS & RHESSI \\
\hline 1 Multi-Pitch Rotating Modulator & 9 Rotating Modulation Collimators (RMC) \\
\hline Single grid & Each RMC has two identical grids \\
\hline Continuous range of grid pitches & Uniform grid pitch in each RMC \\
\hline $\begin{array}{l}\text { Single grid samples a quasi-continuous range of source } \\
\text { angular scales }\end{array}$ & $\begin{array}{c}\text { Each RMC samples one angular scale. Requires multiple } \\
\text { RMCs to sample several discrete angular scales }\end{array}$ \\
\hline Detector can spatially resolve finest grid pitch & $\begin{array}{c}\text { Detector has no spatial resolution. Measures total flux } \\
\text { transmitted by grid pair }\end{array}$ \\
\hline Signal at each location is time-modulated & Detector-averaged signal is time-modulated \\
\hline Modulation has quasi-rectangular profile & Modulation has a quasi-triangular profile \\
\hline $\begin{array}{c}\text { Achievable angular resolution limited by detector } \\
\text { resolution }\end{array}$ & Achievable angular resolution limited by grid pitch \\
\hline Grid uniformity is unimportant, provided it is known & Grid uniformity is critical to performance \\
\hline No requirement on grid orientation provided it is known & $\begin{array}{l}\text { Maintaining relative orientation of two grids is critical } \\
\text { to performance }\end{array}$ \\
\hline
\end{tabular}

For opacity at $2.2 \mathrm{MeV}$, the grid is fabricated from $2.5-\mathrm{cm}$ thick tungsten/copper slats using a technique similar to that of the RHESSI coarse grids. The grid is composed of 65 modules (Figure 7), where each module has a fixed pitch (Figure 8). The total grid mass is $\sim 50 \mathrm{~kg}$. Unlike the case of RHESSI grids, however, the imaging performance relies on knowledge of the slat locations, not their exact placement, a feature that simplifies grid fabrication.

Since the imaging technique is based on the timing and location of individual photon interactions, imaging analysis can be applied post facto to any desired subset of detected events such as those restricted to a particular energy range or polarization signature. As a result, the spectroscopic and polarization properties of the detector are fully compatible with the imaging so that the system functions as an imaging polarimetric spectrometer.

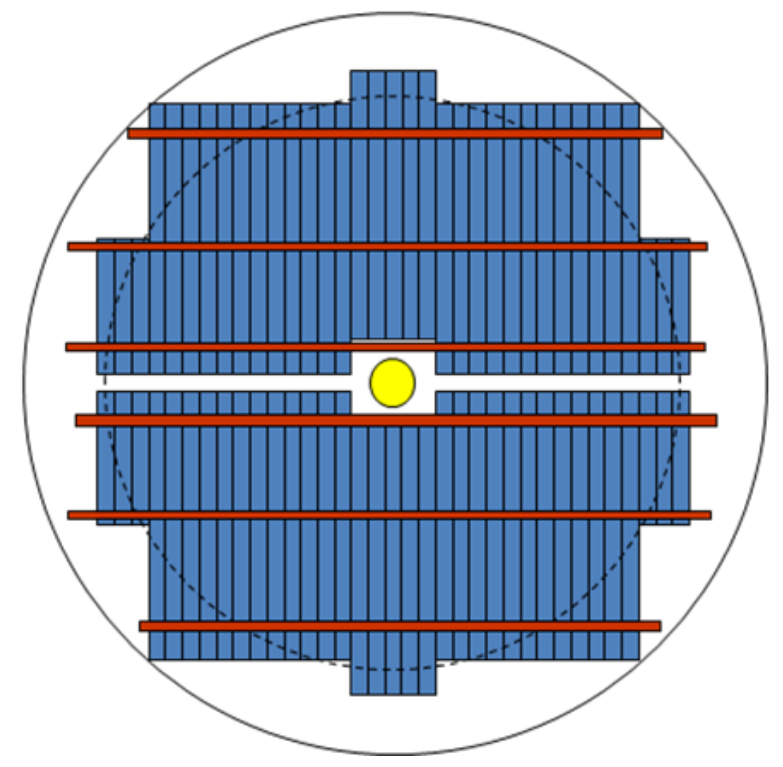

Figure 7. Schematic view of the MPRM (44-cm diameter inscribed circle, 57-cm diameter circumscribing circle), with the 3 -cm diameter lens (yellow) for the aspect system. The bundles of parallel slats (blue rectangles) span 13 different pitches. 


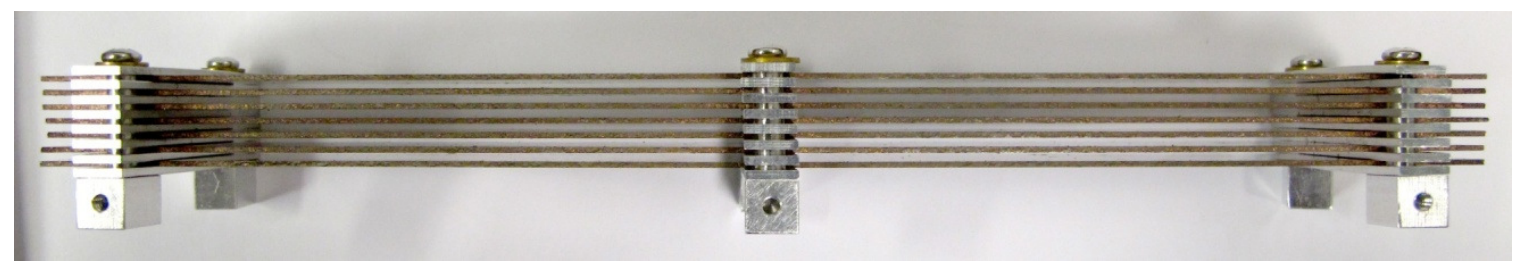

Figure 8 . Photo of the initial, test bundle (13-mm wide) with the median pitch $(7 \mathrm{~mm})$.

\subsection{Imager aspect system}

The GRIPS imager aspect system plays three roles: first, it provides post-facto high cadence relative aspect to enable imaging with 12.5 -arcsecond resolution to be done with a telescope pointed to only \pm 0.5 degrees; second, it provides absolute aspect knowledge to permit the resulting images to be placed on the Sun to arcsecond accuracy; third, it provides the information by which flexure and twist of the 8-m boom can be fully compensated during data analysis, thereby easing the mechanical requirements of the boom itself. There are three components to the aspect system: pitch/yaw knowledge, relative-roll knowledge, and absolute-roll knowledge.

The pitch/yaw aspect system uses a filtered lens, integrally mounted in the center of the rotating grid (see Figure 7). The lens focuses a white-light solar image on an X-ray transparent, diffuse-reflecting screen fixed to the front of the detector system (Figure 9). The location of the image (inferred from its sharp limbs) establishes the offset between the detectorgrid and Sun-detector lines of sight and so can be used to fully compensate for pointing variations or mechanical flexure of the boom. A $2048 \times 2048$ boom-mounted CCD camera monitors the locations of the solar image relative to fiducials on the screen. A plastic cover over the boom minimizes scattered light on the screen. The use of the reflected images in this context allows optimization of the measured image scale ( 6 arcseconds or $0.25 \mathrm{~mm} / \mathrm{pixel})$ while the fiducials eliminate pointing and stability requirements on the camera. Scaling from the RHESSI solar aspect system (SAS), relative aspect accuracies of $\sim 2$ arcseconds can be expected. Onboard processing identifies the subset of pixels needed by ground-based software to determine the positions of 4 limbs relative to the fiducials.

The relative-roll aspect system uses small holes within the rim of the rotating grid. These holes provide "point sources" that are continuously observed by a second $2048 \times 2048$ CCD camera, viewing the grid from its mount on the detector assembly, to provide post-facto knowledge of the relative orientation of the grid and detector system. As with the pitch/yaw aspect system, onboard processing identifies the subset of pixels needed by ground-based software to determine the positions of two point sources. Note that this knowledge of the relative orientation of the grid relative to the detector axes eliminates the need for indexing or accurate speed control of the rotation drive and also eliminates any structural twist requirement on the boom.

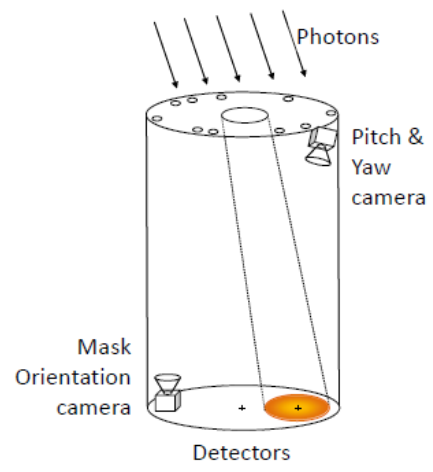

Figure 9. Schematic illustrating the pitch/yaw aspect system and the relative-roll aspect system. The absolute-roll aspect system is not shown.

The absolute-roll aspect system uses reflective prisms to view the horizon in opposite directions by a single, third CCD camera mounted on the gondola. The use of a single camera avoid systematic errors from co-aligning two horizonviewing cameras. This system will correct for the expected pendulation of the payload ( \pm 0.1 degrees), and thus allows the conversion of the observed relative orientation for accurate image placement on the Sun. 


\subsection{Support systems}

The GRIPS gondola and rotor (Figure 10) are designed to accommodate and control the 8-m boom. The boom will be oriented horizontally for launch as previously done with other balloon payloads with long booms. The gondola serves as the main support for the entire system: the GRIPS telescope (spectrometer/polarimeter plus MPRM grid), the electronics bays, and the CSBF Science Interface Package (SIP). The gondola structure is constructed of bolted aircraft-grade aluminum, and is designed for both azimuthal and elevation pointing without obstructing the FOV of the instrument.

The gondola is designed to withstand the shocks associated with cut down and impact, and to protect the instrument with crush pads during landing and possible rollover. The grid end of the telescope and solar arrays are designed to be expendable and are expected to be replaced after each flight. Crush pads are attached to the bottom and sides for energy absorption during landings. The mass of the science gondola (excluding ballast and SIP) will be $\sim 3500 \mathrm{lbs}$.
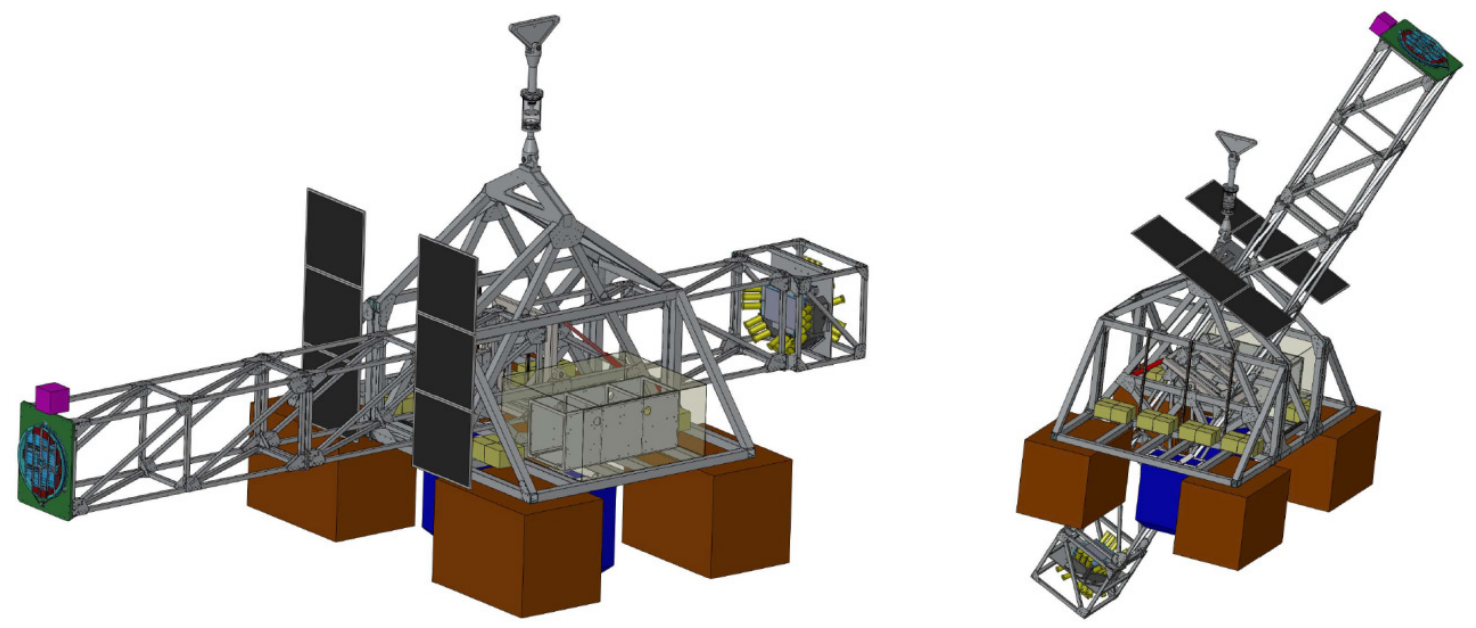

Figure 10. Schematics of the gondola with the boom oriented horizontally for launch/landing (left) or raised in elevation to point at the Sun (right).

Azimuth control is accomplished by means of a servo motor controlled rotor in series between the gondola and the balloon train. The telescope will be capable of pointing from the horizon to an elevation of 65 degrees. Above this elevation, the inflated balloon can occult the Sun. Although the balloon would not significantly attenuate X-rays and gamma-rays, the solar aspect system that is used for image reconstruction would be unable to function.

The complete housekeeping stream, and the science data, is recorded by multiple solid-state flash drives, totaling $\sim 200$ GB capacity. The data will be downlinked continuously during line-of-sight through a $400 \mathrm{kB} / \mathrm{s}$ one-way downlink using CSBF-supplied transmitters and receivers. The total continuous data rate for GRIPS, including housekeeping data, is expected to be $\sim 4$ GB per day, scaled up from NCT. The science data consists of the record of individual events in the 3D-GeDs, complete with energy and location information. Onboard processing will produce quicklook lightcurves and spectra, which will be telemetered through the Iridium communications network throughout the balloon flight. If available, the CSBF-supplied TDRSS connection (up to $115.2 \mathrm{kbps}$ ) will allow for downlinking of a subset of the unprocessed data. The bulk of the data will be stored on the solid-state flash drives, to be recovered after each flight.

Total power use for the full instrument and gondola is estimated to be $\sim 500 \mathrm{~W}$. The continental-US engineering test flight will be powered entirely from batteries, with no need for solar panels. For the Antarctic LDBFs, $5 \mathrm{~m}^{2}$ solar panels will be used to power the gondola and to charge a battery for backup power. 


\section{DATA ANALYSIS}

Analyzing the measured data of a Compton telescope is a non-trivial task due to the large, sparsely-sampled data space opened up by high-resolution detectors and the large amount of measured parameters compared to the small amount of detected events. Fortunately, much of the existing software developed for NCT and RHESSI can be easily adapted for GRIPS. The GRIPS data analysis consists of: 1) depth and energy calibration, 2) event reconstruction, 3) event selection, and 4) subsequent imaging, spectroscopic, or polarization analysis.

In the first step, "depth and energy calibration", the measured A/D converter units, timings, and detector strip IDs are converted into positions and energies. While determining the calibration matrices, etc. is a time consuming process, the software itself will be identical to that developed for the $N C T$ calibrations ${ }^{27}$. The second step, "event reconstruction", transforms measured positions and energies of individual interactions (Compton scattering, photoelectric effect, etc.) into the photon scattering sequence allowing the recovery of the photon energy and incident direction, while also identifying and rejecting background events. The interaction sequence is determined by Compton kinematics and scatter probabilities (Figure 11). Three different methods have been developed for this task ${ }^{23,28}$ : the classic approach, the Bayesian method, and a neural network approach. Since all approaches are optimized for astrophysics (multiple sources at unknown position on high background), implementing optimizations for solar physics (single source at known position) is expected to further improve the performance of these methods.

During the third step, "event selections", those Compton events most likely to have originated from the Sun are identified. The knowledge of the energy of the recoil electron and of the energy and direction of the scattered gamma ray restricts the origin of the gamma ray to a Compton cone. This allows selecting only those events whose cone intersects the position of the Sun and thus greatly reduces atmospheric background. Additional cuts on Compton scatter angles, interaction distances, event quality factors, etc. might help to further minimize the background.

All event reconstruction and event selection methods are implemented in the MEGAlib software package ${ }^{29}$ and are actively used on simulated and measured NCT data. MEGAlib also contains methods for polarization analysis (e.g. Figure 4 has been determined utilizing MEGAlib) but they still need to be optimized for solar physics.

MEGAlib will then provide a list of good events originating from the Sun with the corresponding time, energy, and location in the detector for each photon. Images from the MPRM imaging system can then be constructed by groundbased software using the aspect knowledge and techniques well-established with RHESSI (see Figure 6). Spectra for the selected photons can be obtained using RHESSI software.
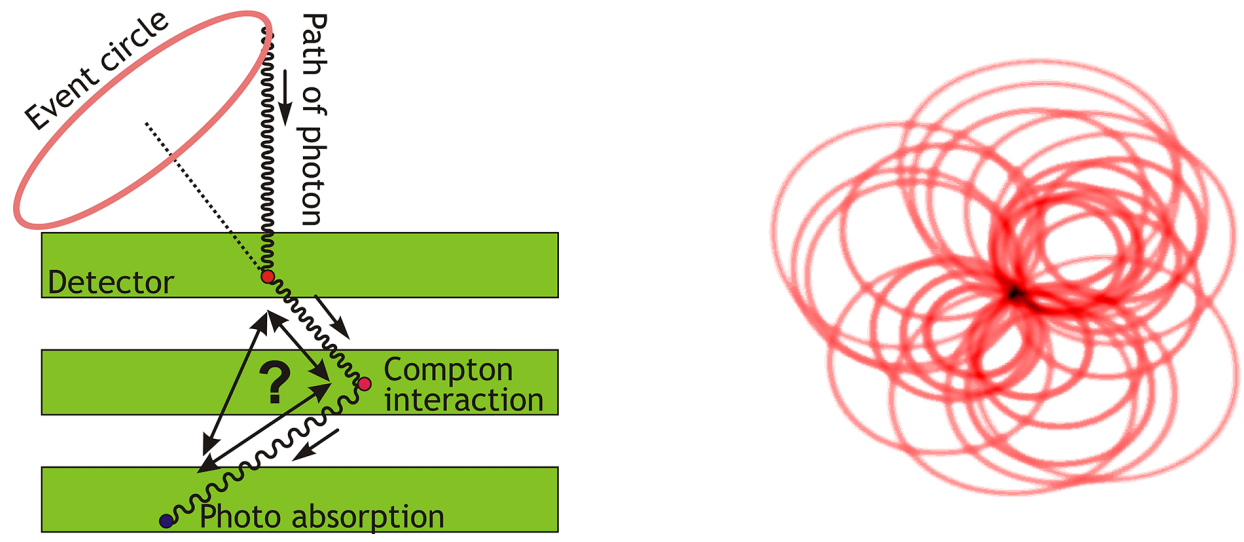

Figure 11. Left: the sequence of interactions in the detector has to be determined utilizing Compton kinematics and scatter probabilities. Right: via Compton imaging techniques, the origin of each gamma ray is restricted to a cone, or a circle on 2D "all sky" projections, and for a point source, those circles overlap at the true source position. Note that Compton imaging is only used to identify photons of likely solar origin; the high-resolution gamma-ray imaging capability of GRIPS is accomplished with the MPRM. 


\section{ANTICIPATED PERFORMANCE}

\subsection{Modeling results}

A GEANT model of the 3D-GeDs (based on real NCT detectors) has been used to simulate the performance of the GRIPS spectrometer/polarimeter. Simulated effective areas at various energies are in Table 1. To make an image in a given energy band, a minimum of $\sim 200$ counts are needed ${ }^{7}$, although many more counts are needed to take full advantage of the excellent point-response function of the MPRM.

With respect to polarimetry, the model predicts a modulation factor of $45 \%$ for $100 \%$ linearly polarized photons in the energy range $150-650 \mathrm{keV}$. The relation between the total bremsstrahlung $>300 \mathrm{keV}$ fluence (used to facilitate later comparisons) from a flare and the minimum detectable polarization (MDP) at the three-sigma level is calculated with the following assumptions: flare duration of 600 seconds, photon spectral index of 3, and a typical Antarctic background that results in a background count rate of 47 counts/sec after event selections (Figure 12).

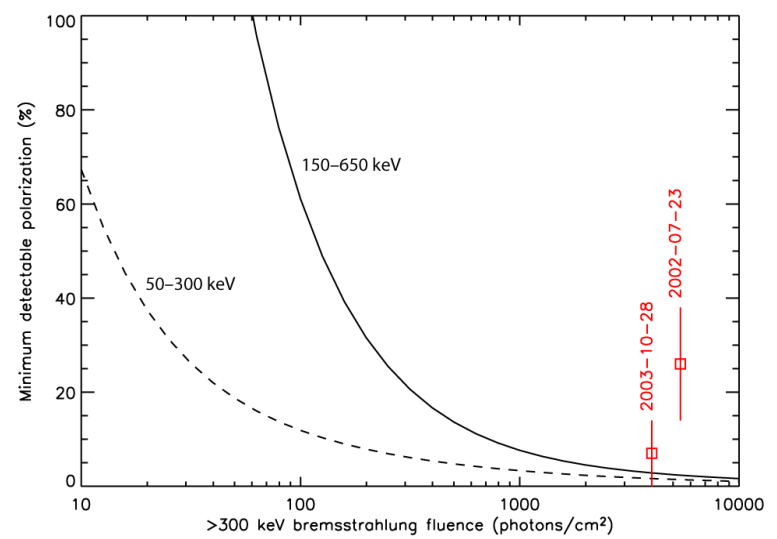

Figure 12. The predicted minimum detectable polarization (MDP, $3 \sigma$ ) in two energy ranges versus the total bremsstrahlung fluence above $>300 \mathrm{keV}$ : $150-650 \mathrm{keV}$ observed by the 3D-GeDs (solid line), and 50-300 keV (dashed line) observed by an already-built $\mathrm{Si} / \mathrm{CdTe}$ polarimeter that is not part of the GRIPS instrument but may be a piggyback instrument on the gondola. The red data points show the large uncertainties for RHESSI observations of gamma-ray polarization (200-400 $\mathrm{keV}$ ) for two flares ${ }^{4}$.

\subsection{Long-duration balloon flights (LDBFs)}

Following its engineering test flight in fall 2013, the GRIPS instrument will be completed with its full complement of 3D-GeDs and a solar power system in preparation for long-duration balloon flights (LDBFs) from Antarctica. The first possible opportunity would be the Antarctic summer starting December 2014, which allows for around-the-clock observation of the Sun. An alternative would be Ultra-LDBFs, although the super-pressure balloons currently being developed would not yet be able to carry a payload as heavy as GRIPS to the necessary altitudes. These would allow launches anytime in the year, and flights of up to 100 days or more. Thus, GRIPS could be stored and launched when solar activity increased, say, due to a large active region coming over the east limb.

The likelihood of observing a flare with gamma-ray emission can be estimated by using the long-term observations of the RHESSI ${ }^{9}$ and the Gamma Ray Spectrometer (GRS) on $S M M^{30}$. Flare occurrence with respect to time is used to predict the performance of GRIPS to account for the non-Poisson nature of flare events: an active region that produces one gamma-ray flare is likely to produce others as well. Consequently, this clustering of events results in a decreased chance (relative to Poisson statistics) of observing at least one gamma-ray flare, yet if one is seen, then there is an increased chance of seeing multiple gamma-ray flares.

The SMM/GRS and RHESSI data sets were restricted to periods in the same phase of the solar cycle as 2013-15, and sliding window functions were used to simulate observations by balloon flights (14-day or 28-day). We note that the largest gamma-ray flares of the last maximum occurred in Oct-Nov 2003 and Jan 2005, 11 years before the LDBFs planned here. From SMM and RHESSI observations, Shih et al. ${ }^{9}$ showed that the $2.223 \mathrm{MeV}$ line fluence has a close linear correlation to the observed $>300 \mathrm{keV}$ bremsstrahlung hard X-ray fluence. Figure 13 shows the probability of seeing at least one flare above a given threshold $>300$ bremsstrahlung fluence based on the flare statistics from the peaks of solar cycles 21 and 22. Producing an image of neutron-capture line emission would require at minimum 200 counts. 
Combining this number with the computed effective area and the previously mentioned correlation result, a flare would need to emit an equivalent $>300 \mathrm{keV}$ bremsstrahlung fluence of at least $\sim 180$ photons $/ \mathrm{cm}^{2}$ (red, dotted line in Figure 13). Thus, for one 28 -day flight, we predict a $>\sim 40 \%$ chance of observing a flare that can be imaged in this line, and for a flight each year for two years, we estimate a $>\sim 65 \%$ chance.

A flare of this size has an MDP of 35\% and, with an assumed spectral index of 3, has $~ 5,000$ photons $/ \mathrm{cm}^{2}$ in the 50-100 $\mathrm{keV}$ band, or $\sim 70,000$ counts total. The MPRM would be able to produce hard X-ray images of excellent quality. Of course, smaller, and correspondingly more common, hard X-ray flares would also be detected and imaged.

The likelihood of detecting polarization is indicated by comparing the plot of MDP (three-sigma) versus bremsstrahlung fluence with the probability plot from SMM statistics (Figures 12 and 13). On a 28-day flight, there is a 33\% chance of observing a flare with at least $\sim 330$ photons $/ \mathrm{cm}^{2}$ bremsstrahlung fluence, which corresponds to an MDP of $20 \%$ at 150 $650 \mathrm{keV}$.

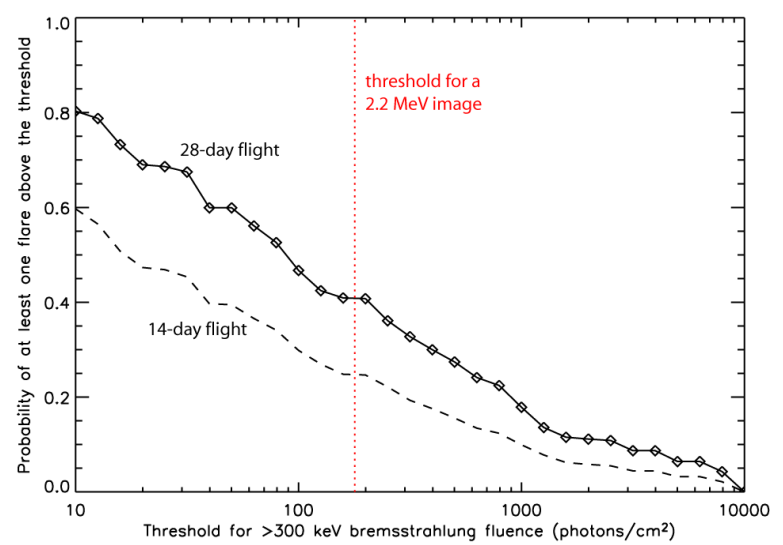

Figure 13. The predicted probability of observing at least one flare on an LDBF above a given threshold of $>300 \mathrm{keV}$ bremsstrahlung emission (solid black line for a 28-day flight, dashed black line for a 14-day flight). The red, dotted line denotes the approximate minimum bremsstrahlung emission corresponding to a flare that can be imaged in the neutroncapture line.

\section{CONCLUSIONS}

The balloon-borne GRIPS instrument is the next step in studying high-energy ion and electron acceleration in solar flares. The observation of one or more hard X-ray flares on the 1-day engineering test flight (fall 2013) will validate the utilized approach. Although solar activity is difficult to predict, Antarctic LDBFs have a decent chance of observing a flare that can be imaged in the neutron-capture line. In addition to the science return from these flights, the balloon fights will prove the technologies used by GRIPS.

The ideal platform for a GRIPS-type instrument is on a spacecraft. A realistic space GRIPS instrument could have sixtyfour 3D-GeDs (four layers in a 4×4 grid) with a MPRM grid on a 20-meter or longer boom (such booms have already been flight qualified) on a rotating spacecraft. The MPRM imaging design does not require precise pointing or stability on the boom, since the solar aspect system can provide precise knowledge of the instantaneous location of the MPRM grid. Such a space GRIPS would provide a tremendous leap forward for high-energy solar physics, providing order-ofmagnitude increases of effective area for gamma-ray line (and hard X-ray) imaging, with much better angular resolution and image quality (Table 1). The need for such observations is expected to be highlighted in the forthcoming Heliophysics Decadal Survey.

\section{ACKNOWLEDGEMENTS}

This effort is funded in part by NASA grant NNX08BA28G. R. P. Lin is supported in part by the WCU grant (No. R3110016) funded by the Korean Ministry of Education, Science, and Technology. Graduate students and a postdoctoral researcher have also been supported in part by fellowships through the NASA Graduate Student Researchers Program and the NASA Posdoctoral Program, respectively. 


\section{REFERENCES}

[1] Lin, R. P., et al., "RHESSI observations of particle acceleration and energy release in an intense solar gamma-ray line flare," ApJ 595, L69-L76 (2003)

[2] Ramaty, R. and Manzhavidze, N., "Gamma-rays from solar flares," IAU Symposium 195, 123-132 (2000)

[3] Lin, R. P., et al., "The Reuven Ramaty High-Energy Solar Spectroscopic Imager (RHESSI)," Sol. Phys. 210, 3-32 (2002)

[4] Boggs, S. E., Coburn, W. and Kalemci, E., "Gamma-ray polarimetery of two X-class solar flares," ApJ 638, 1129 1139 (2006)

[5] Ramaty, R. and Murphy, R. J., "Nuclear processes and accelerated particles in solar flares," Space Sci. Rev. 45, $213-$ 268 (1987)

[6] Smith, D. M., et al., "High-resolution spectroscopy of gamma-ray lines from the X-class solar flare of 2002 July 23," ApJ 595, L81-L84 (2003)

[7] Hurford, G. J., et al., "First gamma-ray images of a solar flare," ApJ 595, L77-L80 (2003)

[8] Hurford, G. J., et al., "Gamma-ray imaging of the 2003 October/November solar flares," ApJ 644, L93-L96 (2006)

[9] Shih, A. Y., Lin, R. P. and Smith, D. M., "RHESSI observations of the proportional acceleration of relativistic $>0.3$ $\mathrm{MeV}$ electrons and $>30 \mathrm{MeV}$ protons in solar flares," ApJ 698, L152-L157 (2009)

[10] Share, G. H., et al., "RHESSI $\mathrm{e}^{+}-\mathrm{e}^{-}$annihilation radiation observations: implications for conditions in the flaring solar chromospheres," ApJ 615, L169-L172 (2004)

[11] Krucker, S., et al., "Coronal gamma-ray bremsstrahlung from solar flare-accelerated electrons," ApJ 678, L63-L66 (2008)

[12] McConnell, M. L., et al., "Hard X-ray solar flare polarimetry with RHESSI," Adv. Space. Res. 34, 462-466 (2004)

[13] McConnell, M. L., et al., in preparation

[14] Lei, F., Dean, A. J. and Hills, A. G., "Compton polarimetry in gamma-ray astronomy," Space Sci. Rev. 82, 309-388 (1997)

[15] Chanan, G., Emslie, A. G. and Novick, R., "Prospects for solar flare X-ray polarimetry," Sol. Phys. 118, 309-319 (1988)

[16] Suarez-Garcia, E., et al., "X-ray polarization of solar flares measured with RHESSI," Sol. Phys. 239, 149-172 (2006)

[17] Chang, H.-K., Boggs, S. and Chang, Y.-H., "The Nuclear Compton Telescope (NCT): Scientific goals and expected sensitivity," Adv. Space Res. 40, 1281-1287 (2007)

[18] Amman, M. and Luke, P. N., "Three-dimensional position sensing and field shaping in orthogonal-strip germanium gamma-ray detectors," Nucl. Inst. Meth. A 452, 155-166 (2000)

[19] Amrose, S., et al., "Calibration of 3D positioning in a Ge cross-strip detector," Nucl. Inst. Meth. A 505, 170-173 (2003)

[20] Luke, P. N., et al., "Amorphous Ge bipolar blocking contacts on Ge detectors," IEEE Trans. Nucl. Sci. 39, 590-594 (1992)

[21] Luke, P. N., et al., "140-element Ge detector fabricated with amorphous Ge blocking contacts," IEEE Trans. Nucl. Sci. 41, 976-978 (1994)

[22] Coburn, W., et al., "3D positioning germanium detectors for gamma-ray astronomy," Proc. SPIE 4784, 54-63 (2003)

[23] Zoglauer, A., "First light for the next generation of Compton and pair telescopes," Ph.D. dissertation, Technical University Munich (2005)

[24] Boggs, S. E. and Jean, P., "Performance characteristics of high resolution Compton telescopes," A\&A 376, 11261134 (2001)

[25] Tajima, H. et al., "Design and performance of the soft gamma-ray detector for the NeXT mission," IEEE Trans. Nucl. Sci. 52, 2749-2757 (2005)

[26] Hurford, G. J., et al., “The RHESSI imaging concept,” Sol. Phys., 210, 61-86 (2002)

[27] Bowen, J.D., et al., "Pre-flight calibration of the prototype Nuclear Compton Telescope," Proc. SPIE 6266, 626625 (2006)

[28] Zoglauer, A., et al., "Recognition of Compton scattering patterns in advanced Compton telescopes," Proc. SPIE 6700, 67000I (2007)

[29]Zoglauer, A., Andritschke, R. and Schopper, F., "MEGAlib The Medium Energy Gamma-ray Astronomy Library," New Astr. Rev. 50, 629-632 (2006)

[30] Vestrand, T., et al., "The Solar Maximum Mission atlas of gamma-ray flares," ApJS 120, 409-467 (1999) 